THU0330

PREVALENCE OF RAYNAUD'S PHENOMENONIN THE NORTHERN PARTS OF THE NETHERLANDS: AN EPIDEMIOLOGICAL STUDY OF THE LIFELINES COHORT

Amaal Eman Abdulle ${ }^{1}$, Elisabeth Brouwer ${ }^{2}$, Harry van Goor ${ }^{3}$, Anniek van Roon ${ }^{1}$, Johanna Westra ${ }^{2}$, Karina de Leeuw ${ }^{2}$, Douwe J Mulder ${ }^{1} .{ }^{1}$ University of Groningen University Medical Center Groningen, Department of Internal Medicine, division Vascular Medicine, Groningen, Netherlands; ${ }^{2}$ University of Groningen - University Medical Center Groningen, Department of Rheumatology and Clinical Immunology, Groningen, Netherlands; ${ }^{3}$ University of Groningen - University Medical Center Groningen, Department of Pathology and Medical Biology, Groningen, Netherlands

Background: Although several previously conducted studies reported on the prevalence of Raynaud's phenomenon (RP) in different regions of the world, these studies often included a limited number of selected individuals. Moreover, no studies exist that have systematically assessed the relative contribution of known etiological factors of RP in the general population of the Netherlands.

Objectives: To assess the prevalence of RP, and gender-specific etiological factors associated with RP in the Northern parts of the Netherlands. Methods: Data from the Lifelines cohort were analyzed, in which all participants completed the self-administered validated connective tissue disease questionnaire. Subjects who reported cold-sensitive fingers and bior triphasic colour changes in response to cold were considered to suffer from RP. Known etiological factors such as hormonal status, body mass index (BMI), smoking behaviour, and comorbidities were all assessed in a standardised way.

Results: In total 93935 participants completed the questionnaire (mean age $45.6 \pm 12.9$ ). The prevalence of RP was $4.2 \%\left[\begin{array}{lll}95 \% & \mathrm{Cl} & 4.1-4.4\end{array}\right]$ which was approximately three-fold higher in females $(5.7 \%, 95 \% \mathrm{Cl}[5.5-$ $5.9])$, as compared to males $(2.1 \%, 95 \% \mathrm{Cl}$ [1.9-2.2], p-value $<0.001$, figure 1). Regarding gender-specific risk factors associated with RP, we observed that $\mathrm{BMI}<18.5$ (OR 4.6 [2.4-8.7], $\mathrm{p}<0.001$ ), cardiovascular disease (OR $1.53[95 \% \mathrm{Cl} 1.31-1.78], \mathrm{p}<0.001$ ), history of cancer (OR 1.40 [1.00-1.95], $p=0.049$ ), use of beta-blockers (OR 1.39 [1.06-1.83], $p=0.01$ ), and smoking (OR 1.28 [1.09-1.51], $\mathrm{p}=0.003$ ) were associated with an increased odds of RP in men. Conversely, alcohol consumption, diabetes and age were not associated with RP in men. In females, BMl<18.5 (OR 2.9 [2.27-3.64], $\mathrm{p}<0.001$ ), cardiovascular disease (OR 1.42 [1.32-1.54], $p<0.001$ ), receiving hormonal contraception (OR 1.17 [1.08-1.26], $p<0.001$ ), and hormonal replacement therapy (OR 1.14 [1.04-1.25], $\mathrm{p}=0.007$ ) were associated with increased odds of RP. Moreover, smoking behavior, use of beta-blockers, alcohol consumption, and diabetes were not associated with RP in women. A BMl $>30$ was associated with a strongly decreased odds of RP in both men (OR 0.22 [0.11-0.42], $\mathrm{p}<0.001)$ and women (OR 0.35 [0.28-0.44], $\mathrm{p}<0.001)$.

Conclusion: This large cohort study found a prevalence of $4.2 \%$ of RP in the Northern part of the Netherlands, with an expected predominance in young female subjects. Moreover, the etiologic risk factors of RP are multifactorial and clearly gender-specific (e.g., hormonal status in women, smoking behavior and use of beta-blockers in men), with underweight strongly increasing and obesity strongly decreasing the likelihood of RP in both sexes. This might suggest that different mechanisms influence the expression of RP in men and women.

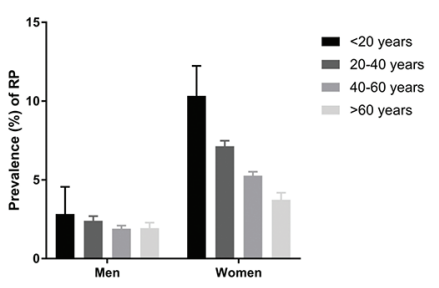

Abstract THU0330 - Figure 1. Prevalence of RP depicted for men and women.

Disclosure of Interests: Amaal Eman Abdulle : None declared, Elisabeth Brouwer Speakers bureau: Dr. Brouwer as an employee of the UMCG received speaker fees and consulting fees from Roche which were paid to the UMCG, Harry van Goor: None declared, Anniek van Roon: None declared, Johanna Westra: None declared, Karina de Leeuw: None declared, Douwe J Mulder Grant/research support from: My University has received research grants for my research from: Boehringer Ingelheim and Actelion, Speakers bureau: My University has received speakers fee from: Sanofi

DOI: 10.1136/annrheumdis-2019-eular.4005

\section{THU0331 \\ SKELETAL TROPONIN I A POSSIBLE NOVEL BIOMARKER FOR MANAGEMENT OF PATIENTS WITH IDIOPATHIC INFLAMMATORY MYOPATHIES}

Katriina Bamberg ${ }^{1}$, Laura Mehtälä ${ }^{1}$, Olli Arola ${ }^{2}$, Seppo Laitinen ${ }^{3}$, Pauliina Nordling ${ }^{4}$, Marjatta Strandberg ${ }^{4}$, Niko Strandberg ${ }^{5}$, Johanna Paltta ${ }^{6}$ Markku Mali' ${ }^{6}$, Fabricio Espinosa-Ortega ${ }^{7,7}$, Laura Pirilä ${ }^{7}$, Ingrid E. Lundberg ${ }^{7}$ Tanja Savukoski ${ }^{1}$, Kim Pettersson ${ }^{1} .{ }^{1}$ University of Turku, Department of Biochemistry/Biotechnology, Turku, Finland; ${ }^{2}$ Turku University Hospital, Intensive Care Medicine and Pain Management, Turku, Finland; ${ }^{3}$ Regional Laboratory Nordlab Kajaani, Kajaani, Finland; ${ }^{4}$ Turku University Central Hospital, Heart Center, Turku, Finland; ${ }^{5}$ Turku University Central Hospital, Department of Orthopaedic Surgery, Turku, Finland; ${ }^{6}$ Turku University Central Hospital, Department of Rheumatology, Turku, Finland; ${ }^{7}$ Karolinska Institute, Division of Rheumatology, Department of Medicine, Stockholm, Sweden

Background: The current biomarkers for the diagnosis and monitoring of diseased and injured skeletal muscles, such as creatine kinase (CK), have limited tissue specificity and incapability to differentiate between pathological and physiological changes. Thus, new biomarkers with improved diagnostic certainty are needed. Skeletal troponin I (skTnl) is a promising new biomarker for injured and diseased skeletal muscle tissue. Although studies have reported that circulating skTnl levels are elevated in response to trauma, exercise and various muscular diseases (1), its clinical utility to serve as a diagnostic indicator has largely been unexplored.

Objectives: Our aim was to develop and validate a novel assay for skTnl, and to assess its clinical performance with idiopathic inflammatory myopathy (IIM) patients.

Methods: A two-step fluoroimmunoassay was used to analyze the levels of skTnl in samples from healthy reference individuals $(n=125)$, trauma patients $(n=151)$, and patients with IIM $(n=94)$. Later, skTnl and CK levels in patients with IIM were compared according to their disease activity status (active, pre-active or stable)

Results: The limit of detection was $1.2 \mathrm{ng} / \mathrm{ml}$, and the upper reference limit (90th percentile) was $5.4 \mathrm{ng} / \mathrm{ml}$. The median skTnl concentrations were $<\mathrm{LOD}, \quad 2.7 \mathrm{ng} / \mathrm{ml}$, and $9.8 \mathrm{ng} / \mathrm{ml}$ in reference, trauma, and IIM cohorts, respectively. Differences in measured skTnl levels were statistically significant between all three study cohorts (Mann-Whitney $\mathrm{p}<0.001$ for all). skTnl and $\mathrm{CK}$ had a strong positive correlation (Spearman's $r=0.848, p<0.001$ ). With skTnl, patients in both pre-active and active IIM were differentiated from stable phase patients $(33.9$ and $34.5 \mathrm{ng} / \mathrm{ml} \mathrm{vs}$ $5.1 \mathrm{ng} / \mathrm{ml}, \quad p<0.001$ for both). This was not possible with $\mathrm{CK}$ as significantly elevated CK levels were mainly present in active IIM (median 16.5 $\mu k a t / L)$ and the medians of pre-active and stable phase patients (4.8 $\mu k a t / L$ vs $1.7 \mu k a t / L, \quad p=0.060$ ) remained close to normal reference ranges. The area under the receiver operator characteristic curve was $0.87,0.84$, and 0.87 for skTnl and CK individually and combined, respectively.

Conclusion: With the developed skTnl assay, IIM patients were identified from healthy individuals and from patients with traumatic muscular injuries. Also, skTnl was shown to outperform CK in detecting IIM patients in different disease activity statuses.

\section{REFERENCE:}

[1] Kiely PDW, Bruckner FE. Serum skeletal troponin I in inflammatory muscle disease: Relation to creatine kinase, CKMB and cardiac troponin I. Ann Rheum Dis 2000;59:750-1.

Disclosure of Interests: Katriina Bamberg: None declared, Laura Mehtälä: None declared, Olli Arola: None declared, Seppo Laitinen: None declared, Pauliina Nordling: None declared, Marjatta Strandberg: None declared, Niko Strandberg: None declared, Johanna Paltta: None declared, Markku Mali: None declared, Fabricio Espinosa-Ortega: None declared, Laura Pirilä Consultant for: Abbvie, Novartis, Eli Lilly, MSD Finland, Pfizer, UCB, Roche, Jansen- Cilag, Sandoz, Speakers bureau: Pfizer, Ingrid E. Lundberg Grant/research support from: Dr. Lundberg has received honoraria from Bristol Myers Squibb and Medlmmune and is currently receiving a research grant from Bristol Myers Squibb and from Astra Zeneca., Consultant for: She is a scientific advisor for Bristol Myers Squibb, and aTyr, Tanja Savukoski: None declared, Kim Pettersson: None declared DOI: 10.1136/annrheumdis-2019-eular.7436 